\title{
A compound figure of merit for photonic applications of metal nanocomposites
}

\author{
Hernando Garcia \\ Department of Physics Southern Illinois University Edwardsville, IL 62026 \\ Hare Krishna and Ramki Kalyanaraman \\ Department of Physics Washington University in St. Louis St. Louis, MO 63130
}

\begin{abstract}
Selecting nanocomposites for photonic switching applications requires optimizing their thermal, nonlinear and two-photon absorption characteristics. We simplify this step by defining a compound figure of merit $\left(F O M_{C}\right)$ for nanocomposites of noble metals in dielectric based on criteria that limit these structures in photonic applications, i.e. thermal heating and two-photon absorption. The device independent results predict extremely large values of $F O M_{C}$ for a specific combination of the metal and insulator dielectric constant given by $\epsilon_{h}=\frac{\epsilon_{1}-\epsilon_{2}}{2}$, where $\epsilon_{h}$ is the dielectric constant of the host and $\epsilon_{1}$ and $\epsilon_{2}$ are the real and imaginary parts for the metal.
\end{abstract}


In recent years considerable research has gone into the study of all-optical switching devices for photonic applications where the requirement of the material had been a large $n_{2}$ (nonlinear refractive index), low index change due to thermal effects, and small two-photon absorption (TPA) [1, 2]. Furthermore, there is significant interest in using nanocomposites comprising of metal nanoparticles in dielectric host for such applications because of their nonlinear response [3, 4, 5] and because of their potential use in applications below the diffraction limited regime [6, 7, 8]. However, the selection of the metal-host system for a given application must be based on a careful evaluation of the key physical limitations in the interaction of light with matter. In this regard, there are two well accepted limitations. The first is the refractive index change due to thermal effects arising from light-matter interactions [1]. The second, which has been recognized as the ultimate limit for optical switching, is TPA [9]. However, there has been no effort to combine these effects into a figure of merit describing the metal nanocomposite material in photonic applications involving optical modulation [10].

In this letter we define a compound figure of merit $\left(F O M_{C}\right)$ as the product of the thermal FOM $\left(F O M_{T h e r m}\right)$ and the TPA FOM $\left(F O M_{T P A}\right)$. The reason for choosing the product as compared to a linear combination comes from the strong correlation between the TPA and thermal effects. In general material having large TPA show small linear absorption and thermal effects and vice versa. Therefore it is difficult to select materials for such applications based simply on the linear combination of the two effects. A common example of such a definition is that of the gain-bandwidth product used to optimize an op-amp. the The theoretical behavior for $\mathrm{Ag}, \mathrm{Au}$ and $\mathrm{Cu}$ nanoparticles embedded in an $\mathrm{SiO}_{2}$ matrix were evaluated based on this FOM. The behavior of the $F O M_{C}$, which is device independent, shows that an extremely large value results at a specific combinations of the metal and host dielectric function given by $\epsilon_{h}=\frac{\epsilon_{1}-\epsilon_{2}}{2}$, where $\epsilon_{h}$ is the dielectric constant of the host and $\epsilon_{1}$ and $\epsilon_{2}$ are the real and imaginary parts of the the dielectric constant of the metal. These results could simplify the process of materials selection for nanocomposites in photonic switching applications at desired wavelengths.

We start with the criteria that conventional all-optical switching devices are based on generation of light induced phase shifts of the order of $p \pi$, where $4 \geq p \geq 1$ for a range of devices. The design in mind for these calculations is a Mach-Zehnder interferometer where $p=1$, and the criterion for switching is given by [7]:

$$
p \pi=\frac{2 \pi n_{2} I_{o} L}{\lambda}
$$

where $I_{o}$ is the switching intensity, $L$ is the length of the device, and $\lambda$ is the wavelength. Our first figure of merit requires that the thermal index change be a small fraction of the fast light-induced change $\left(n_{2} I_{o}\right)$ 
[1]. The thermal index change can be expressed by:

$$
\text { thermal index change }=\frac{\partial n}{\partial T} \frac{\Delta Q}{C_{p} \rho V}
$$

where $\frac{\partial n}{\partial T}$ is the thermo-optic coefficient, $C_{p}$ is the specific heat, $V$ is the volume of the material heated by the light, $\rho$ is the density of the material and $\Delta Q$ is the energy absorbed by the material per pulse, which is given by:

$$
\Delta Q=\alpha L I_{O} A \tau
$$

where $A$ is the cross sectional area of the device, $\alpha$ is the linear absorption coefficient and $\tau$ is the duration of the switching pulse. By using eq. 2 and 3 the $F O M_{\text {Therm }}$ becomes:

$$
F O M_{\text {Therm }}=\frac{n_{2} C_{p} \rho}{\frac{\partial n}{\partial T} \tau \alpha}
$$

The behavior of this FOM for $\mathrm{Au}, \mathrm{Ag}$ and $\mathrm{Cu}$ nanoparticles embedded in $\mathrm{SiO}_{2}$ is shown in Fig. पa). The values of various parameters are listed in tables $\llbracket$ and $\amalg$

Two-photon absorption can reduce the device throughput even if the linear absorption is small. A general criterion for this was developed by Stegeman and co-workers [2] based on Eq. 1 and the two photon absorption coefficient $\beta$ as:

$$
\frac{\beta \lambda}{2 n_{2}}<1 \text { or } \frac{2 n_{2}}{\beta \lambda}>1
$$

Based on this, we defined our next figure of merit to be:

$$
F O M_{T P A}=\frac{2 n_{2}}{\beta \lambda}
$$

The behavior of this quantity is shown in Fig. 1 (b) for the noble metal nanoparticles.

The compound figure of merit in this letter takes into account the switching limitations due to thermal heating and large two photon absorption and is defined as the product of Eq. 4 and Eq. 5]to give:

$$
F O M_{C}=F O M_{\text {Therm }} . F O M_{T P A}=\frac{2 n_{2}^{2} C_{p} \rho}{\alpha \tau \beta \lambda \frac{\partial n}{\partial T}}
$$

Using this definition, an attractive combination of metal-dielectric would be one with a large value of the $F O M_{C}$. It is natural to introduce this figure of merit because there could be materials with excellent thermal properties but very large TPA, or vice versa, or various other combinations, including both high or both low. On the other hand materials showing a trade off between moderate TPA and moderate thermal heating may be excellent candidates for photonic applications where the only limitations will be device design and optimization. 
Using this new $F O M_{C}$, we evaluated the response for metal nanocomposites. In these structures we have a lot of freedom in choosing and controlling $\alpha, \beta$, and $n_{2}$, which are the only free parameters in the above figure of merit. In the evaluation we assumed the following:

1. $\rho, C_{p}$, and $\frac{\partial n}{\partial T}$ can be considered to be properties of the host only and are not influenced by the presence of the metal nanostructures (i.e. dilute limit approximation);

2. $\beta, \alpha$, and $n_{2}$ are drastically modified by dielectric confinement, and they may be analyzed within the context of Mie theory [11];

3. The particle diameters are such that quantum confinement effects due to intra-band dipole electronic transitions are negligible;

4. The main contribution to the nonlinear susceptibility comes from the inter-band electric dipole transition, and the contribution is negative, and purely imaginary.

With the above assumptions we calculated $F O M_{C}$ (Eq. 6) for a metal nanocomposite, by noting the following:

$$
\frac{n_{2}}{\beta \lambda}=\frac{\operatorname{Re}\left[\chi_{e f f}^{(3)}\right]}{4 \pi \operatorname{Im}\left[\chi_{e f f}^{(3)}\right]}
$$

where we have ignored, for the moment, the frequency dependence of the nonlinear susceptibilities. $\chi_{e f f}^{(3)}$ is the effective nonlinear susceptibility and is given in the context of Mie's theory by:

$$
\chi_{e f f}^{(3)}=v f_{1}^{2}\left|f_{1}\right|^{2} \chi_{m}^{(3)}
$$

where $v$ is the volume fraction of the metal in the host matrix, $\chi_{m}^{(3)}$ is the third order susceptibility of the metal cluster, and $f_{1}$ is an enhancement factor produced by the dielectric confinement, which can be expressed as:

$$
f_{1}=\frac{3 \epsilon_{h}}{\epsilon_{1}+i \epsilon_{2}+2 \epsilon_{h}}
$$

where $\epsilon_{h}$ is the real dielectric constant of the host, and $\epsilon_{1}$ and $\epsilon_{2}$ are the real and imaginary dielectric constants of the metal nanoparticles respectively. Using Eq. 8 in Eq. 7] and using assumption (4) we get for the ratio of the real and imaginary parts in Eq. 7 .

$$
\frac{\operatorname{Re}\left[\chi_{e f f}^{(3)}\right]}{\operatorname{Im}\left[\chi_{e f f}^{(3)}\right]}=-\frac{\operatorname{Im}\left[f_{1}^{2}\right]}{\operatorname{Re}\left[f_{1}^{2}\right]}
$$


We calculated the ratio of $n_{2}[12]$ and $\alpha$ [4], using the following definitions:

$$
\alpha=\frac{v \omega\left|f_{1}\right|^{2} \epsilon_{2}}{n_{o} c}
$$

and

$$
n_{2}=\frac{12 \pi}{n_{o}^{2} c} \operatorname{Re}\left[\chi_{e f f}^{(3)}\right]=\frac{12 \pi}{n_{o}^{2} c} v\left|f_{1}\right|^{2} \operatorname{Re}\left[f_{1}^{2} \chi_{m}^{(3)}\right]
$$

where, $n_{o}$ is the refractive index, $c$ is the velocity of light and $\omega$ is the frequency of the incident light. This results in a ratio of:

$$
\frac{n_{2}}{\alpha}=\frac{6 \lambda\left|\chi_{m}^{(3)}\right|}{n_{o} c \epsilon_{2}} \operatorname{Re}\left[i f_{1}^{2}\right]
$$

By using the above result and Eq. 9 in Eq. 6 we get the compound FOM as:

$$
F O M_{C}=\frac{108 C_{p} \rho\left|\chi_{m}^{2}\right|}{\pi \tau \frac{\partial n}{\partial T} n_{o} c}\left\{\frac{\lambda \epsilon_{2} \epsilon_{h}^{2}\left(\epsilon_{1}+2 \epsilon_{h}\right)^{2}}{\left[\left(\epsilon_{1}+2 \epsilon_{h}\right)^{2}-\epsilon_{2}^{2}\right]\left[\left(\epsilon_{1}+2 \epsilon_{h}\right)^{2}+\epsilon_{2}^{2}\right]^{2}}\right\}
$$

The dependence of the $F O M_{C}$ on the dielectric constants has been put in the brackets. As would be expected, this $F O M_{C}$ goes to zero under the highly absorbing conditions found at the plasmon resonance condition of $\epsilon_{1}+2 \epsilon_{h}=0$. On the other hand, as Fig. 1(c), large maxima are observed in the wavelength range of 0.3 to $0.6 \mu \mathrm{m}$ for the various metals using the parameters from table $\llbracket$ The large maxima occur at the condition of:

$$
\left(\epsilon_{1}+2 \epsilon_{h}\right)^{2}-\epsilon_{2}^{2}=0
$$

The wavelengths corresponding to these maxima for each metal are tabulated in table III] and are compared to nearby peak positions in the $F O M_{T h e r m}$ and $F O M_{T P A}$. A significant difference in wavelength of the $F O M_{C}$ to those in the thermal or TPA cases is observed. This difference is especially important when put in the context of data communication bandwidths, where wavelength separations of 1 to $5 \mathrm{~nm}$ are routine. The physical origin of these wavelengths can be understood by analyzing the location of the peaks within the framework of the simplifying Drude model. In this model the real and imaginary components of the dielectric susceptibility of the metal in the high frequency limit $(\omega \tau>>1)$ are given by:

$$
\epsilon_{1}=\epsilon_{o}-\frac{\omega_{p}^{2}}{\omega^{2}} \text { and } \epsilon_{2}=\frac{\omega_{p}^{2}}{\omega^{2} \tau}
$$

where $\epsilon_{o}$ is the contribution to the dielectric constant of the metal from bound charges and $\omega_{p}$ is the bulk plasmon resonance of the metal. Using the condition for the $F O M_{C}$ maxima and the condition for surface plasmon resonance given by:

$$
\frac{\omega^{2}}{\omega_{s p}^{2}}=\epsilon_{1}+2 \epsilon_{h}
$$


After some algebraic manipulation, the frequency $\omega_{F O M}$ at which the maxima in the $F O M_{C}$ occurs can be related to the surface plasmon frequency $\omega_{s p}$ by the expression:

$$
\left(\frac{1}{\omega_{s p}^{2}}-\frac{1}{\omega_{F O M}^{2}}\right)^{2}=\left(\frac{1}{\omega_{F O M}^{3} \tau}\right)^{2}
$$

In eq. 15. the quantity on the right side is always positive, but the quantity on the left side can be positive or negative giving:

$$
\frac{\omega_{F O M}^{2}}{\omega_{s p}^{2}}=\left(1 \pm \frac{1}{\omega_{F O M} \tau}\right)
$$

Therefore we find that the maxima in the $F O M_{C}$ can be located above or below the surface plasmon resonance. In the evaluation of eq. 14 we have chosen the positive values obtained from the above expres-

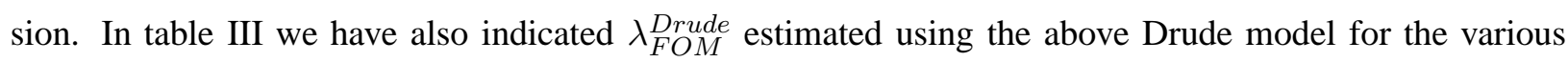
metals using experimental values for $\lambda_{s p}$ and the relaxation times from Ref. [13]. It is apparent that this crude model also predicts a shift in the peak position from $\lambda_{s p}, \lambda_{T h e r m}$ and $\lambda_{T P A}$ and actually predicts very well the $\lambda_{F O M}$ position for the case of $\mathrm{Cu}$ estimated from Eq. 14

Recently there have been reports of lasing of Au nanoparticles in water [14]. The authors estimated a surface plasmon resonance for their Au-water system to be at $700 \mathrm{~nm}$ while the observed lasing occurred with various peaks located at $720, \mathrm{~nm}$ or higher. Using our model, we have estimated that the $F O M_{C}$ achieves a maximum for Au nanoparticles in water at $720 \mathrm{~nm}$ for an electron relaxation time of $9.3 \times 10^{-5} \mathrm{~s}$. While this may be rather coincidental, it should be emphasized that the $F O M_{C}$ expressed by eq. 14 represents a situation where losses are minimized and the nanoparticles act as excellent scattering centers.

In conclusion, we have defined a compound figure of merit $F O M_{C}$ for metal-dielectric nanocomposites that takes into account limitations to optical switching from thermal index changes and two photon absorption. As expected the $F O M_{C}$ is zero at the plasmon resonance when large absorption occurs but has extremely large values at a value of frequency which is dependent only upon the dielectric constants of the metal and dielectric. This result could allow the rapid selection of a metal-host system for operation at a desired wavelength.

One of the authors (RK) acknowledges support by the National Science Foundation through grant \# DMI-0449258. 
[1] S. R. Friberg and P. W. Smith, IEEE J Quant. Elect. 23, 2089 (1987).

[2] G. I. Stegeman, E. M. Wright, N. Finlayson, R. Zanonic, and C. T. Seaton, J. Lightwave. Tech. 6, 953 (1988).

[3] L. Yang, Ph.D. thesis, Vanderbilt University (1993).

[4] L. Yang, D. H. Osborne, R. F. Haglund Jr, R. H. Magruder, C. W. White, R. A. Zuhr, and H. Hosono, Appl. Phys. A 62, 403 (1996).

[5] W. T. Wang, Z. H. Chen, G. Yang, D. Y. Guan, G. Z. Yang, Y. L. Zhou, and H. B. Lu, Applied Physics Letters 83, 1983 (2003).

[6] R. F. Haglund Jr, Mat. Sci. Eng. 253, 275 (1988).

[7] S. Maier, P. Kik, H. Atwater, S. Meltzer, E. Harel, B. Koel, and A. Requicha, Nature Materials 2, 229 (2003).

[8] J. H. Huter, E. Fendler, Adv. Mater. 16, 1685 (2004).

[9] V. Mizrahi, K. W. DeLong, G. I. Stegeman, M. A. Saifi, and M. J. Andrejco, Opt. Lett. 14, 1140 (1989).

[10] E. Hartfield and B. Thompson, Handbook of Optics (McGraw-Hill, 1978), chap. Optical modulators, pp. 17-1 $-17-24$.

[11] M. Born and E. Wolf, Principles of Optics (Pergamon Press, 1980), 6th ed.

[12] R. L. Sutherland, Handbook of Nonlinear Optics (Marcel Dekker, Inc, 1996), pp. 295-384.

[13] P. B. Johnson, Christy, and R. W., Phys. Rev. B 6, 4370 (1972).

[14] J. Kang and J. Khurgin (Optical Society of America, Long Beach, CA, 2006), QTuI2, conference on lasers and electro-optics/quantum electronics and laser science and photonic applications, systems and technologies. 
Table I: Substrate properties used in calculation of the FOM.

\begin{tabular}{|c|c|}
\hline Substrate Parameters & Value \\
\hline \hline Specific heat capacity, $C_{p}\left(\times 10^{6}\right.$ ergs $\left./ g-K\right)$ & 7.4 \\
\hline Density, $\rho\left(g-\mathrm{cm}^{-3}\right)$ & 2.33 \\
\hline Thermo-optic coefficient, $\partial n / \partial T\left(\times 10^{-6} K^{-1}\right)$ & 0.55 \\
\hline Refractive index, $n_{o}$ & 1.46 \\
\hline Host dielectric constant, $\epsilon_{h}$ & 3.82 \\
\hline
\end{tabular}

Table II: Materials properties for the metals used in the calculation of the FOM.

\begin{tabular}{|c|c|c|c|}
\hline Metal Parameters & $\mathrm{Ag}$ & $\mathrm{Au}$ & $\mathrm{Cu}$ \\
\hline \hline Fermi velocity, $v_{F}\left(\times 10^{8} \mathrm{~m} / \mathrm{s}\right)$ & 1.42 & 1.4 & 1.28 \\
\hline Electron density, $\rho_{e}\left(\times 10^{22} \# / \mathrm{cm}^{3}\right)$ & 5.85 & 5.9 & 8.45 \\
\hline Effective mass, $m_{e f f}$ & $0.96 m_{o}$ & $0.99 m_{o}$ & $0.99 m_{o}$ \\
\hline High frequency dielectric constant, $\epsilon$ & 5.3 & 8.6 & 9.3 \\
\hline Radius \& interparticle spacing $(\mathrm{nm})$ & 35,75 & 35,75 & 35,75 \\
\hline
\end{tabular}

Table III: Values of the wavelengths corresponding to the surface plasmon resonance $\left(\lambda_{s p}\right)$ and strongest peaks in the FOM $M_{\text {Therm }}\left(\lambda_{\text {Therm }}\right), F O M_{T P A}\left(\lambda_{T P A}\right)$ and FOM $M_{C}\left(\lambda_{C}\right)$. The position of the strongest peak as predicted by the crude Drude model is expressed as $\lambda_{F O M}^{\text {Drude }}$.

\begin{tabular}{|c|c|c|c|c|c|}
\hline Metal & $\lambda_{s p}(\mathrm{~nm})$ & $\lambda_{\text {Therm }}(\mathrm{nm})$ & $\lambda_{\text {TPA }}(\mathrm{nm})$ & $\lambda_{C}(\mathrm{~nm})$ & $\lambda_{\text {FOM }}^{\text {Drude }}(\mathrm{nm})$ \\
\hline \hline $\mathrm{Ag}$ & 487 & 450 & 472 & 509 & 497 \\
\hline $\mathrm{Au}$ & 552 & 583 & 550 & 591 & 560 \\
\hline $\mathrm{Cu}$ & 591 & 618 & 618 & 607 & 606 \\
\hline
\end{tabular}




\section{Figure Captions}

- Fig. 1. (a) The thermal FOM, FOM Therm for Ag (solid line), Au (dashed line) and Cu (dotted line) embedded in $\mathrm{SiO}_{2}$ matrix as a function of wavelength. The values for the complex dielectric constant were obtained from ref. [13]. The $F O M_{\text {Therm }}$ for Ag (solid line) has been scaled by a factor of 1/50. (b) The two-photon absorption FOM, FOM $M_{T A}$ for $\mathrm{Ag}$, $\mathrm{Au}$ and $\mathrm{Cu}$ in $\mathrm{SiO}_{2}$ as a function of wavelength. (c) Total figure of merit given by Eq. 14 for $\mathrm{Ag}$ (solid line), Au (dashed line) and $\mathrm{Cu}$ (dotted line) embedded in $\mathrm{SiO}_{2}$ matrix. The wavelengths corresponding to the surface plasmon wavelengths are also indicated for each metal. 


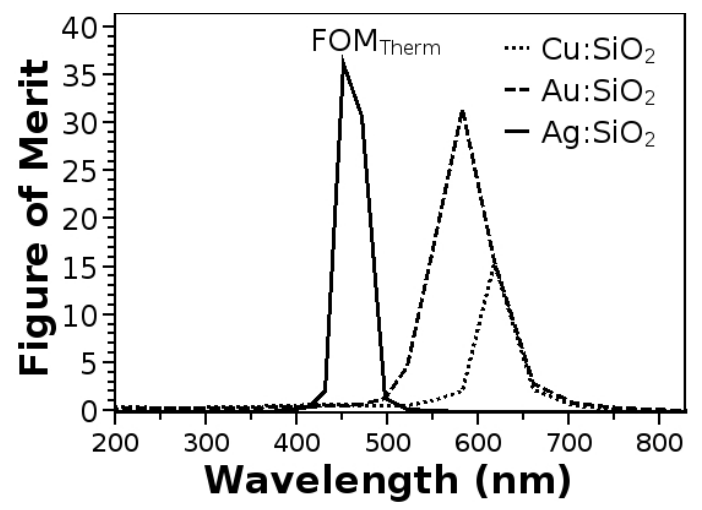

(a)

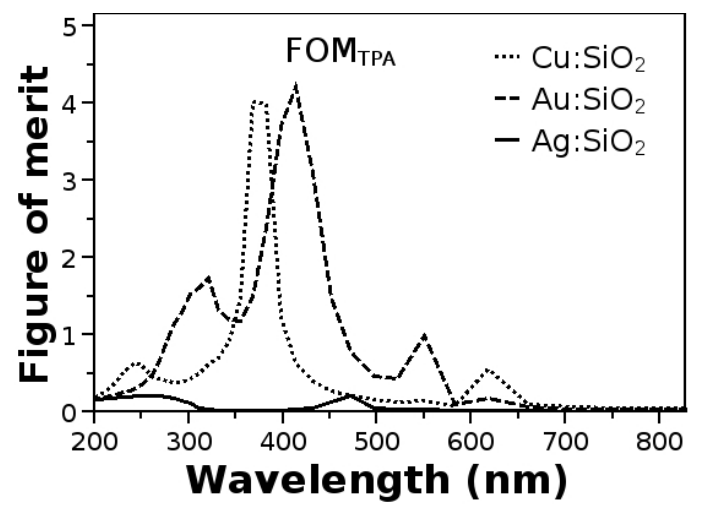

(b)

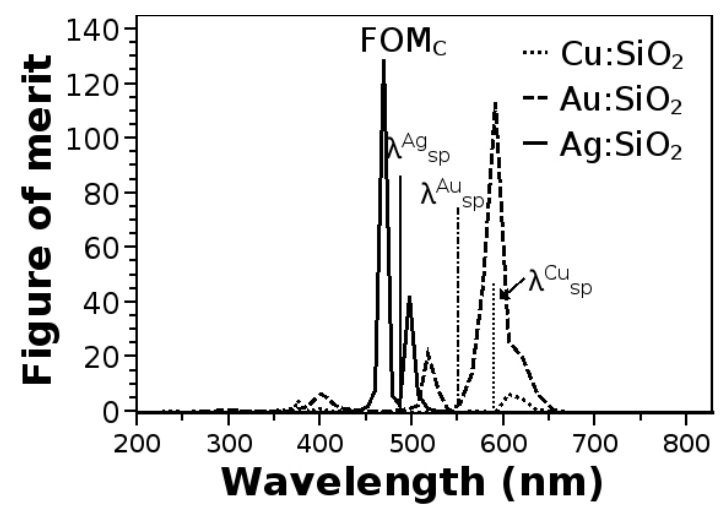

(c)

Figure 1: 